\title{
Phase-Tracking Loop based on Delta-Sigma Oversampling Architecture
}

\author{
Yuichiro Orino and Minoru Kuribayashi Kurosawa \\ Interdisciplinary Graduate School of Science and Engineering \\ Tokyo Institute of Technology, Japan, \\ Email: orino@ae.titech.ac.jp and mkur@ip.titech.ac.jp
}

\author{
Takashi Katagiri \\ Sutekina Inc., Japan, \\ Email: takashi.katagiri@primemotion.com
}

\begin{abstract}
This paper presents a new oversampling architecture for implementing phase-tracking loop that is commonly utilized for position sensors such that synchro, resolver, and incremental encoder. This architecture consists of the cascade connection of three stage: coarse-quantizing and oversampling modulation, direct signal processing, and decimation filtering. It is expected that the oversampling strategy and the signal processing increase the resolution of detecting phase as well as oversampling $\mathrm{A} / \mathrm{D}$ converters. This paper shows an simplest design of the signal processing circuit and some simulation results.
\end{abstract}

\section{INTRODUCTION}

From some position sensors, such like resolver and encoder, position information can be available as not explicit signals but only modulated signals. This causes that auxiliary signal processing is needed to get explicit information prior to further positional processing. For the cases of resolver and encoder etc., there are two algorithms to detect a phase, which means a cyclic positional information, from two-tone incoming signals. One is known as a phase encoding method, which converts incoming signals to digital codes and looks up a map of tanh function. Another way, about which this paper focuses, is to use a phase-tracking loop. Due to recent advances in digital technology, not only phase encoding but also phase-tracking loop can be realized by means of digital signal processing, but the digital phase-tracking loop generally requires complex and large circuits.

This paper presents a new digital architecture for a phasetracking loop for purpose of relaxing circuit requirements on analog and digital components. We employ an unconventional method for signal processing, which is called 'signal processing in sigma-delta (delta-sigma) domain' [1].

\section{PhASE-TRACKING LOOP}

\section{A. Basic Principle}

Figure 1 shows an ideal phase-tracking loop. It is a signal processing system based on feedback loop as like phase-locked loop. Input signals $u_{s}(t), u_{c}(t)$ are generated with original phase information $\theta(t)$, and these are written as:

$$
u_{s}(t)=A \sin \theta(t), \quad u_{c}(t)=A \cos \theta(t)
$$

where $A$ is the amplitude of incoming signals. In general, $\theta(t)$ is continuous function of time $t$.

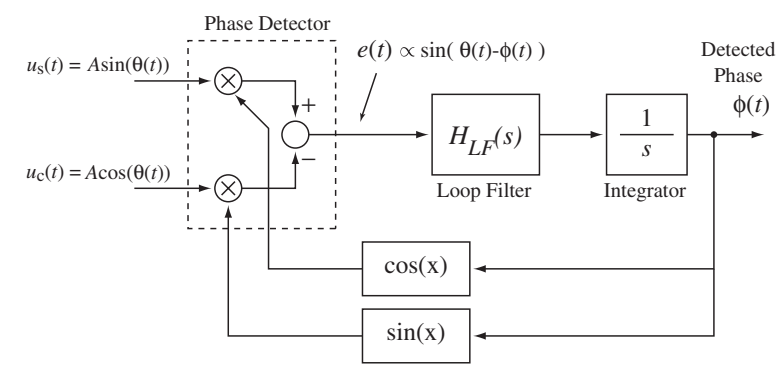

Fig. 1. The basic structure of phase-tracking loop

The phase-tracking loop has an internal phase $\phi(t)$, which also means the output signal of the loop. With the incoming signals and this internal phase, the phase detector calculates a phase error $e(t)$ as following equations:

$$
\begin{aligned}
e(t) & =A \sin \theta(t) \cos \phi(t)-A \cos \theta(t) \sin \phi(t) \\
& =A \sin (\theta(t)-\phi(t))
\end{aligned}
$$

When the deviation of the phase $\theta(t)-\phi(t)$ is enough smaller than $1, e(t)$ can be approximated as:

$$
e(t) \simeq A(\theta(t)-\phi(t))
$$

By means of this error signal $e(t)$ and proper loop filter, which acts as a compensator, the internal phase $\phi(t)$ in the integrator can be controlled to track the original phase $\theta(t)$ because of the effects of negative feedback loop.

\section{B. Realization Methods}

As mentioned above, the phase-tracking loop have been used in front-end circuits for certain position sensors, i.e. synchros, resolvers and incremental encoders, to detect mechanical angular and position. To output the detected information in digital form, like analog-to-digital (A/D) converters do, is also an important role for these circuits. For instance, resolver-todigital converters and interpolators are examples of this type of circuits. There are many variation

Conventionally, front-end circuits based on the phasetracking loop are realized as mixed analog-digital integrated circuits, which work with intense interaction between analog and digital part. Figure 2 shows an example of the conventional system. In such a way, it is generally difficult to obtain both high-accuracy detection and high-rate tracking. But now, due 


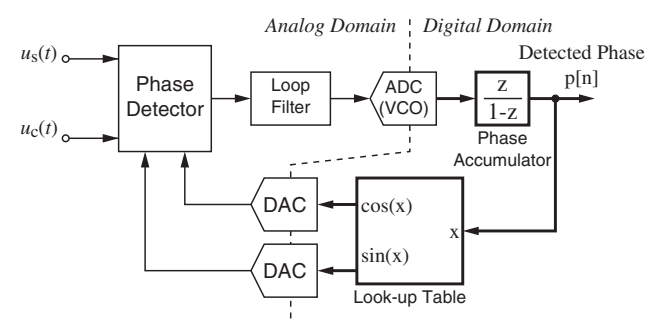

Fig. 2. Conventional phase-tracking loop.

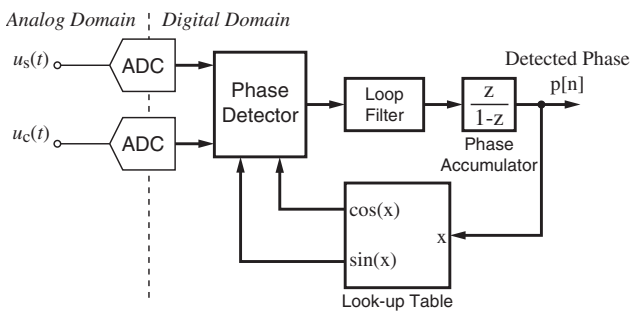

Fig. 3. Cascade-connected digital phase-tracking loop.

to the progress of integrated circuits technology in recent years, it becomes easy to realize a signal processing system based on the phase-tracking loop as a cascade of A/D converters and a digital circuit that works as a phase-tracking loop. An example diagram is shown in Fig. 3.

This cascade architecture is relatively easy to realize and tune than conventional ones. But, in general, higher-resolution and higher-speed calculation capabilities are required for digital signal processing circuits in order to get better performance such as accuracy and tracking rate. High-resolution A/D converters and multi-bit arithmetic circuits, especially multiplier and look-up table, increase total circuit size and cost.

This paper presents a new signal processing architecture for realizing the phase-tracking loop. In this architecture, we employs oversampling technique and delta-sigma modulation in order to decrease the complexity of analog and digital circuits.

\section{ARCHITECTURE OF PROCESSING SYSTEM}

The basic techniques of the proposed architecture are oversampling and delta-sigma (sigma-delta) modulation. Oversampling is a useful technique for converting signals between analog and digital formats. The definition of oversampling is simply to sample at a higher frequency than Nyquist rate, but it allows high-resolution representation as a result of anti-alias filtering and decimation from oversampling rate to Nyquist rate.

On the other hand, delta-sigma modulation is a technique for controlling quantization noise. To use delta-sigma modulations together with the oversampling technique is very efficient because delta-sigma modulations suppress in-band quantization noise even with coarse quantization.In another words, deltasigma modulations can trade resolution-in-time for resolutionin-amplitude.

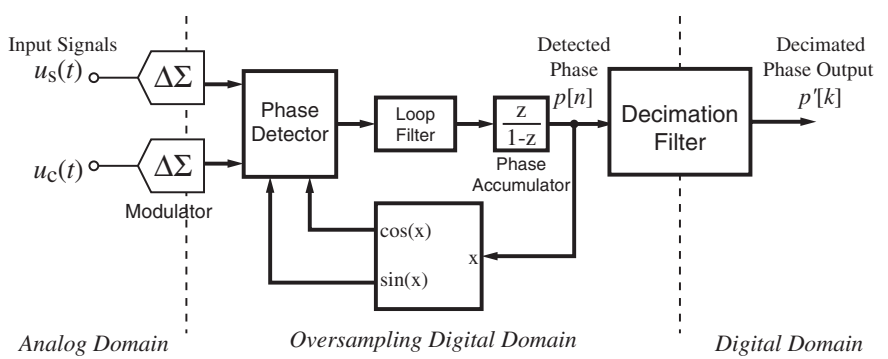

Fig. 4. A schematic diagram of the proposed architecture for phase-tracking loop

Because of relaxed requirements for analog components, oversampling converters, which are often called delta-sigma $\mathrm{A} / \mathrm{D}$ and $\mathrm{D} / \mathrm{A}$ converters, have become popular in recent years for these applications that require high-resolution conversion within a relatively narrow frequency band, especially in system-on-chip designs.

The architecture utilized in this paper for realizing phasetracking loop is based on the oversampling and delta-sigma modulating strategy. The heart of this architecture is to directly process digital signals, which are sampled and modulated by delta-sigma oversampling converters, before decimation. There are some prior works with the same idea for several application, i.e. sensor signal processing [2], control systems [4], communication systems [5], and audio processing [3]. This technique for signal processing is called 'signal processing in sigma-delta domain' [1], or 'bit-stream signal processing' [5] when 1-bit signals are processed. In contrast with earlier works for sensor signal processing, the aim of our work described in this paper is to realize more complex signal processing that requires multipliers and loop.

Figure 4 shows a schematic diagram of the proposed phasetracking system based on this strategy. The system consists of three parts: oversampling delta-sigma modulators, processing circuits for modulated signals, and decimation filter, and these are cascade-connected.

As a general rule, to process at oversampling rate mostly requires faster processing system than that in case of nonoversampling systems. However, with the present silicon integrated-circuit technology, it is not difficult and not impractical to realize as a specific hardware, not as a general processor and software. With coarsely quantized input signals, especially with a single bit, it is expected that the processing circuit will be realized simply in small chip area. Therefore, there will be no potential difficulty in realizing circuits based on the proposed architecture. In addition, considering the ease of realization of delta-sigma modulators in standard CMOS process, the simplicity of total circuit including analog and digital components is also expected, and it would be an advantage as well.

Moreover, there are an expectation of getting better performance, not only of realization merits. It is expected that this architecture allows high-resolution detection after decimation even with coarse resolution of internal phase due to the effect 


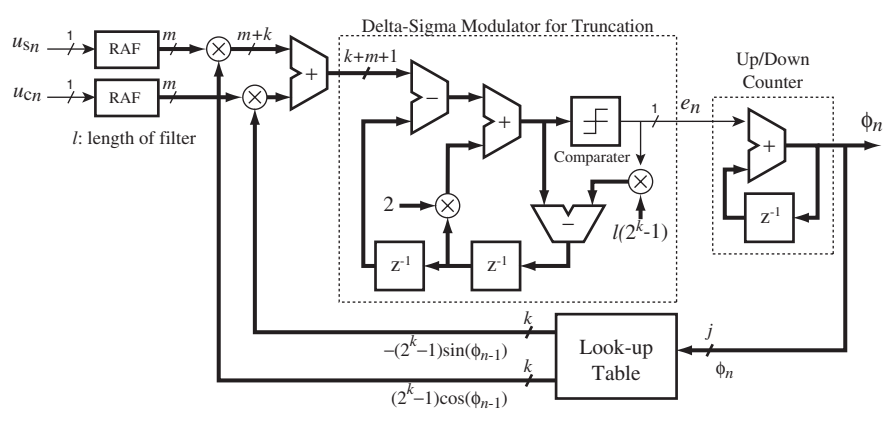

Fig. 5. Circuit diagram of oversampling-rate processing part.

of oversampling. Of coarse, this will be depend on how realize a signal processing circuit. So, in the following parts of this paper, we shows an simple algorithm-level circuit design of phase-tracking loop for the purpose of showing effectiveness of our approach.

\section{Signal Processing Circuit Design}

Figure 5 schematically shows the algorithm-level design of the circuit for phase-tracking loop. We assume the use of 1bit delta-sigma modulators as A/D converters. It consists of several number of adders/subtracters, some registers, and a look-up table. We note that some implicit operations such like bit expansion are not drawn in the figure to make it understandable. The resolution of the internal phase is $j$-bit, and samples in the sine and cosine look-up table that roles sine and cosine functions are provided by $m$-bit fixed-point representations ideally.

One of the most important matters in arithmetic calculations for phase-tracking loop is about multiplying in the phase detection. In proposed circuit design, there are running average filters (RAFs) prior to multipliers. This is because that multiplying delta-sigma signals results in white-like noise [1]. It is necessary to filter out the high frequency spurs of deltasigma modulated signal before multiplying [6]. RAF is most simplest solution.

Each RAFs in the circuit outputs a $m$-bit signal as a result of summing up past $l$ samples. So, each multipliers has to do $m$-bit $\times k$-bit multiplication. The circuit size of these multipliers is a problem. Without RAFs $(m=1, l=1), 1$ bit $\times k$-bit multiplying can be simply realized as a 2-input multiplexor because 1-bit signals represent \pm 1 , however such multiplying might degrade the accuracy of the whole system in exchange for amazing simplicity. In practical, we should deal this incompatibility as a trade-off problem between circuit simplicity and accuracy.

And next, the result of the calculation for phase detection, that is in $k+m+1$ bit, means the deviation for phasetracking feedback loop. In the proposed circuit design, the deviation signal is truncated into 1-bit signal by digital deltasigma modulation. The purpose of this is to keep the following phase accumulation simple. If the modulation for truncation is not used, it is necessary to use a wide bit-width accumulator instead of the simple up/down counter, phase truncation for

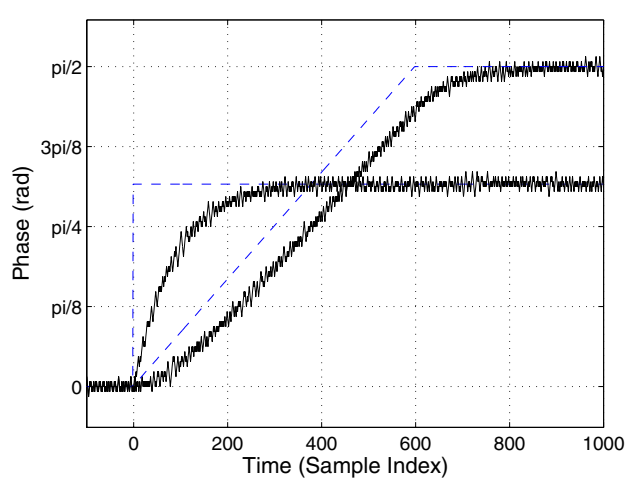

Fig. 6. Time-domain responses of the proposed model without pre-filtering.

looking-up the table, and highly complex filter for decimation because of wider bit-width of phase.

Truncation obviously creates truncation noise. But with delta-sigma modulation, truncation noise is shaped as in the delta-sigma A/D converters. Considering the truncated signal is transmitted into the counter that work as an integrator, at least two or higher order of modulation than two is required for suppressing in-band noise.

\section{Simulations}

Simulations were performed using the computation environment MATLAB to check and evaluate performance of proposed architecture with the algorithm described above. For all simulations in this paper, delta-sigma modulators for A/D conversion were ideally emulated by discrete-time secondorder modulators with dithering, and input signals were also ideally prepared and calculated from given phase source in 64-bit floating-point arithmetics. The amplitude of these sine and cosine signals was $A=0.5$; one half of maximum input level of emulated delta-sigma modulators. The internal phase resolution was $j=8$ bit. For focusing attention to influences from coarsely quantized input signals and internal phase resolution, $m=24$ bit of word length of samples in the look-up table was given.

The decimation filter was not taken in to account. Filter design is left for future research. For analysis, in-band frequency components of signals were just evaluated by discrete Fourier transformation.

\section{A. Tracking Responses}

Most basically, tracking responses against step and ramp inputs of phase were confirmed by simulation results. Examples of time-domain response by the proposed circuit model without pre-filters (RAFs) are shown in Fig. 6. These results show that resulting signal have both high-frequency component and slowly but stable response against source signal that should be detected. High-frequency component is so to say noise, but it is expected to work as a dithering signal for quantization, especially in settling time. This expectation is discussed in the following part. 


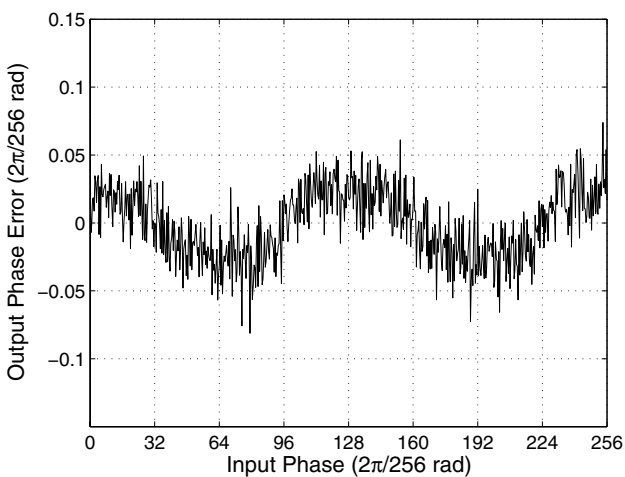

Fig. 7. Detection error for constant-phase inputs by the proposed model without pre-filtering.

\section{B. Settling-Time Accuracy}

We focused on the detection accuracy in settling time. As mentioned above, it is conceptually expected that decimated results can be achieved with fine resolution of phase, as it can not be correctly represented by the quantization levels in the loop. It will be the value and merit of the oversampling architecture. For the purpose of evaluating this concept, simulations for saturated ramp inputs carried out.

Resulting signals in settling time were simply averaged and compared with source phase values. Errors between input values and averaged output values corresponding to it are shown in Fig. 7. The number of averaged samples is $10^{4}$. As a result, while errors have a strong dependency on the phase values, error levels are lower than one half of quantization step (0.5) at whole range of input phase even if input is just a same as quantization levels in the loop or not. This means that it is possible to determine with fine resolution of phase over internal resolution of the phase-tracking loop when decimation filtering is applied with appropriate oversampling ratio (OSR).

Figure 8 show the characteristics of remained in-band noise after decimation at several OSRs. OSR is defined by the rate of decimated signal $f_{0}$ and sampling frequency $f_{s}$ as:

$$
\text { OSR }=\frac{f_{s}}{2 f_{0}}
$$

From the figure 8 , we can see that oversampling ratios of 8 and more result in smaller errors than ideal level of 8-bit quantization at the same rate as oversampling. This means that the oversampling architecture just allows to enhance the conclusive resolution of detected phase.

However, the performance of the noise reduction compared against oversampling ratio is not efficient. This performance saturation is due to the presence of large quantization noise in 1-bit delta-sigma modulated signals and the use of multiplying it without filtering as mentioned above. Figure 9 shows some results of the proposed model with pre-filtering by RAFs. From Fig. 9, we can see that performance improvements are achieved by using filters of at least six of length or higher. More detail analysis of the trade-off problem between circuit simplicity and accuracy is left for future work.

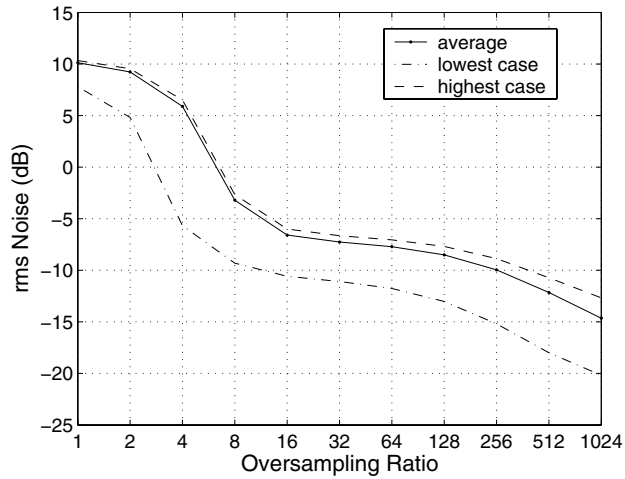

Fig. 8. In-band noise of detected phase by the proposed model without pre-filtering. $0 \mathrm{~dB}$ means the level of ideal 8-bit quantization noise level of $\Delta / 12$.

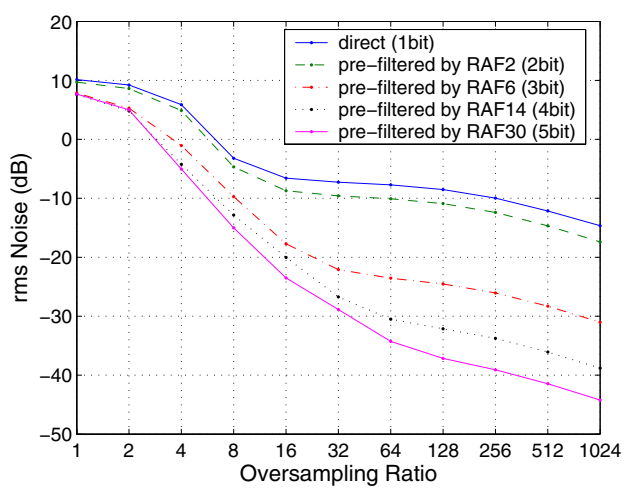

Fig. 9. In-band noise characteristics for oversampling ratios with pre-filtering.

\section{CONCLUSION}

This paper presents a new oversampling architecture for implementing phase-tracking loop that is commonly utilized for position sensors such that synchro, resolver, and incremental encoder. As a result of simulation analyses for example circuit design of digital signal processing, it is expected that the oversampling strategy and the direct signal processing at oversampling rate allows to increase the resolution of detecting phase as well as oversampling A/D converters.

\section{REFERENCES}

[1] Victor da Fonte Dias, "Signal processing in the sigma-delta domain," Microelectronics Journal, vol. 26, no. 6, pp. 543-562, September 1995.

[2] V. Liberali, P. Malcovati, and F. Maloberti, "Sigma-Delta Modulation and Bit-Stream Processing For Sensor Interfaces," in Proc. of Italian Conference on Sensors and Microsystems, 1996.

[3] Josh Reiss and Mark Sandler, "Digital Audio Effects Applied Directly on a DSD Bitstream," in Proc. of the 7th Conference on Digital Audio Effects (DAFx'04), Naples, Italy, October 5-8, 2004.

[4] T. Fukui, M. Segawa, M. Kurosawa, K. Oka and T. Higuchi, "A control system with single-bit digital signal processing," ICARV96, Singapore, pp.1992-1996, 3-6 Dec., 1996.

[5] Fujisaka H.,Sakamoato M. and Morisue M., "Bit-stream signal processing circuits and their application," IEICE Trans. Fundamentals, vol. E85-A, no. 4, pp. 853-860, 2002.

[6] F. Maloberti, "Non Conventional Signal Processing by the Use of Sigma Delta Technique: A Tutorial Introduction," in Proc. of IEEE ISCAS 1992, San Diego, pp.2645-2648, 1992. 